Min Wang*, Shun Zhang, Hong X. Jiang and Hao H. Zhang

\title{
Green synthesis and structural characterization of novel N1-substituted 3,4-dihydropyrimidin- 2(1H)-ones
}

https://doi.org/10.1515/gps-2018-0074

Received April 8, 2018; accepted August 2, 2018; previously published online November 13, 2018

Abstract: Novel N1-substituted 3,4-dihydropyrimidin$2(1 H)$-one derivatives were synthesized through Biginelli condensation of aromatic aldehydes, $\beta$-ketoesters, and monosubstituted (thio)ureas in the presence of copper methanesulfonate at $90^{\circ} \mathrm{C}$ under solvent-free conditions. The screening of the catalysts showed the copper methanesulfonate was the best. Its catalytic activity remained after three times of use. The products were characterized by IR, ${ }^{1} \mathrm{H}$ NMR, ${ }^{13} \mathrm{C} \mathrm{NMR}$, elemental analysis, and X-ray single crystal diffraction technique. A reasonable reaction mechanism was proposed.

Keywords: Biginelli reaction; catalysis; heterocycles; metal sulfonate.

\section{Introduction}

3,4-Dihydropyrimidin-2(1H)-ones (DHPMs) have special therapeutic properties and pharmacological activity. They can be used as calcium channel agents, antiallergic agents, antihypertensive agents and antagonists, and so on [1-6]. Such derivatives have broad applications in antimicrobial, antiviral, anticancer, sterilization, and other fields [7-9]. Several marine alkaloids containing DHPM core were also found. The most notable betzelladine alkaloids can inhibit the binding of HIV gp120 membrane protein. It is expected to treat AIDS [10]. Therefore, the research for the synthesis of DHPMs draws more and more attention.

In recent decades, scientists discovered that N1-substituted DHPMs have better pharmacological activity [11, 12]. However, little attention was paid to them. Few papers about the synthesis were reported. One of the

\footnotetext{
*Corresponding author: Min Wang, College of Chemistry and Chemical Engineering, Bohai University, Jinzhou 121013, P.R. China, e-mail: minwangszg@hotmail.com

Shun Zhang, Hong X. Jiang and Hao H. Zhang: College of Chemistry and Chemical Engineering, Bohai University, Jinzhou 121013, P.R. China
}

Әopen Access. (cc)B (C) 2019 Walter de Gruyter GmbH, Berlin/Boston most important synthetic methods is the Biginelli reaction. New catalysts [13-20] and the explored new ways [21-24] were reported. Nevertheless, some disadvantages such as the expensive catalysts, complex operations, toxic reagents, and the single structure of the product still existed. Consequently, an efficient and green method to synthesize the title products is worth an exploration.

In this study, the N1-substituted DHPMs were synthesized with aromatic aldehydes, ethyl acetoacetate (methyl acetoacetate), and $\mathrm{N}$-substituted (thio)ureas using copper methanesulfonate $\left[\mathrm{Cu}\left(\mathrm{CH}_{3} \mathrm{SO}_{3}\right)_{2} \cdot 4 \mathrm{H}_{2} \mathrm{O}\right.$, abbreviated as CMS] as a catalyst [Eq. (1)]. The $N$-substituted (thio)ureas include methylurea, ethylurea, $p$-tolylurea, and methylthiourea. This method avoids the use of a toxic solvent and a high cost. It is convenient, effective, and green. Twentyone products were synthesized and many of them are new compounds.
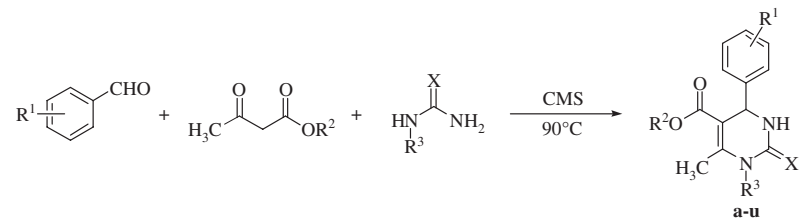

\section{Materials and methods}

\subsection{Materials and general methods}

All reagents were analytic grade and were obtained commercially (Shanghai Chemical Reagent Company, China). CMS was synthesized according to a reported literature [25]. Thin layer chromatography was performed on Merck Silica gel $60 \mathrm{~F}_{254}$ plates using ethyl acetate/ petroleum ether $(\mathrm{V} / \mathrm{V}=3: 7)$ mixture as a mobile phase. Melting points were determined in an open glass capillary on an RY-1 micromelting point apparatus (Tianjin Tianguang Optical Instrument Limited Company, China). A Varian Scimitar 2000 series Fourier transform instrument (Agilent Technologies Inc., Santa Clara, CA, USA) was used for recording the IR spectra using potassium bromide pellets in the range of $400-4000 \mathrm{~cm}^{-1}$. The ${ }^{1} \mathrm{H}$ and ${ }^{13} \mathrm{C}$ NMR spectra were recorded on an Agilent 400-MR instrument (Agilent Technologies Inc., Santa Clara, CA, USA) in DMSO- $d_{6}$ using TMS as an internal standard. Elemental analyses (C, H, N) were conducted using the Elemental Analyser EA 2400II (Perkin Elmer, Waltham, MA, USA), their results were found to be in good agreement $( \pm 0.3 \%)$ with the 
calculated values. Crystallographic data for compound $\mathbf{t}$ were collected on a Bruker Smart Apex II diffractometer (Bruker Corporation, Karlsruhe, Germany) with Mo K $\alpha$ radiation $(\lambda=0.71069 \AA)$ at $296 \mathrm{~K}$ using $\omega$-scan technique. The diffraction data were integrated by using the SAINT program, which was also used for the intensity corrections for the Lorentz and polarization effects. Semiempirical absorption corrections were applied using SADABS program. The structures were solved by direct methods, and all of the non-hydrogen atoms were refined anisotropically on F2 by the full-matrix least-squares technique using the SHELXTL crystallographic software package.

\subsection{General procedure for the synthesis of N1-substituted DHPMs}

Aromatic aldehyde (10 mmol), ethyl acetoacetate (methyl acetoacetate) $(10 \mathrm{mmol}), \mathrm{N}$-substituted urea or a thiourea (13 mmol), and CMS $(0.3 \mathrm{mmol})$ were added in a $25-\mathrm{ml}$ reaction flask. The reaction mixture was then stirred at $90^{\circ} \mathrm{C}$ in an oil bath for an appropriate time (as indicated by TLC). In this process, a solid precipitate was observed in the reaction flask. After completion, the mixture was cooled to room temperature and ice water was added. The crude products were filtrated through a funnel and then purified further by recrystallization with anhydrous ethanol. All title products were characterized by melting point, IR, ${ }^{1} \mathrm{H}$ NMR, ${ }^{13} \mathrm{C}$ NMR, and elemental analysis. The following are spectral data for some of the new compounds:

2.2.1 5-Ethoxycarbonyl-1,6-dimethyl-4-(4-hydroxy-3-methoxyphenyl)-3,4-dihydropyrimidin-2(1H)-one (h): Orange solid. ${ }^{1} \mathrm{H}$ NMR $\left(400 \mathrm{MHz}, \mathrm{DMSO}-d_{6}\right) \delta: 8.94$ (s, $\left.1 \mathrm{H}, \mathrm{OH}\right), 7.87$ (s, $\left.1 \mathrm{H}, \mathrm{NH}\right), 6.60-6.78$ (m, 3H, ArH), 5.08 (s, 1H, CH), 4.06 (q, 2H, J=6.0 Hz, CH $), 3.74$ (s, 3H, $\mathrm{OCH}_{3}$ ), 3.11 (s, 3H, $\mathrm{NCH}_{3}$ ), 2.49 (s, 3H, C=CCH $), 1.15$ (t, 3H, $J=6.0 \mathrm{~Hz}$, $\left.\mathrm{OCH}_{2} \mathrm{CH}_{3}\right) ;{ }^{13} \mathrm{C}$ NMR $\left(100 \mathrm{MHz}\right.$, DMSO- $\left.d_{6}\right) \delta: 166.12,153.65,150.47,147.74$, 146.24, 135.50, 118.50, 115.70, 110.95, 103.32, 59.91, 55.93, 52.46, 30.07, 16.43, 14.56; IR (KBr): 3336, 2968, 1683, 1644, $767 \mathrm{~cm}^{-1}$. Anal. calcd. for $\mathrm{C}_{16} \mathrm{H}_{20} \mathrm{~N}_{2} \mathrm{O}_{5}:$ C 59.99, H 6.29, N 8.74; found C 59.90, H 6.22, N 8.68.

2.2.2 5-Methoxycarbonyl-1,6-dimethyl-4-(4-chlorophenyl)3,4-dihydropyrimidin-2(1H)-one (k): Pale yellow solid. ${ }^{1} \mathrm{H}$ NMR $\left(400 \mathrm{MHz}, \mathrm{DMSO}-d_{6}\right) \delta: 8.07$ (s, $\left.1 \mathrm{H}, \mathrm{NH}\right), 7.39$ (d, $\left.2 \mathrm{H}, J=8.0 \mathrm{~Hz}, \mathrm{ArH}\right)$, 7.26 (d, $2 \mathrm{H}, J=8.0 \mathrm{~Hz}, \mathrm{ArH}), 5.19$ (s, 1H, CH), 3.58 (s, 3H, OCH $), 3.11$ (s, 3H, $\left.\mathrm{NCH}_{3}\right), 2.51$ (s, 3H, $\left.\mathrm{C}=\mathrm{CCH}_{3}\right) ;{ }^{13} \mathrm{C}$ NMR $\left(100 \mathrm{MHz}, \mathrm{DMSO}-d_{6}\right) \delta$ : 166.37, 153.37, 151.72, 143.25, 132.37, 128.89, 128.39, 102.13, 52.14, 51.53, 30.19, 16.51; IR (KBr): 3215, 2950, 1691, 1622, $793 \mathrm{~cm}^{-1}$. Anal. calcd. for $\mathrm{C}_{14} \mathrm{H}_{15} \mathrm{~N}_{2} \mathrm{O}_{3} \mathrm{Cl}$ : C 57.05, H 5.13, N 9.50; found C 56.96, H 5.09, N 9.54.

2.2.3 5-Methoxycarbonyl-1,6-dimethyl-4-(3-hydroxyphenyl)3,4-dihydropyrimidin-2(1H)-one (m): Pale yellow solid. ${ }^{1} \mathrm{H}$ NMR (400 MHz, DMSO-d $) \delta$ : 9.39 (s, 1H, OH), 7.96 (s, 1H, NH), 7.10-6.61 (m, 4H, ArH), 5.08 (s, 1H, CH), 3.58 (s, 3H, OCH $\left.{ }_{3}\right), 3.08$ (s, 3H, NCH $\mathrm{N}_{3}$, 2.47 (s, $\left.3 \mathrm{H}, \mathrm{C}=\mathrm{CCH}_{3}\right) ;{ }^{13} \mathrm{C}$ NMR $\left(100 \mathrm{MHz}\right.$, DMSO- $\left.d_{6}\right) \delta: 166.57,157.84$, 153.67, 151.04, 145.74, 129.88, 116.93, 114.66, 113.25, 102.78, 52.47, 51.54, 30.20, 16.51; IR (KBr): 3377, 2951, 1682, 1598, $787 \mathrm{~cm}^{-1}$. Anal. calcd. for $\mathrm{C}_{14} \mathrm{H}_{16} \mathrm{~N}_{2} \mathrm{O}_{4}: \mathrm{C} 60.86, \mathrm{H}$ 5.84, N 10.14; found C 60.75, H 5.81, N 10.17 .

2.2.4 5-Ethoxycarbonyl-1-ethyl-6-methyl-4-(4-chlorophenyl)-3,4dihydropyrimidin-2(1H)-one (p): White solid. ${ }^{1} \mathrm{H}$ NMR (400 MHz, DMSO- $\left.d_{6}\right) \delta: 7.91(\mathrm{~d}, 1 \mathrm{H}, J=4 \mathrm{~Hz}, \mathrm{NH}), 7.35(\mathrm{~d}, 2 \mathrm{H}, J=8.0 \mathrm{~Hz}, \mathrm{ArH}), 7.19(\mathrm{~d}$, $2 \mathrm{H}, J=8.0 \mathrm{~Hz}, \mathrm{ArH}), 5.10$ (d, 1H, $J=4.0 \mathrm{~Hz}, \mathrm{CH}), 3.98$ (q, $2 \mathrm{H}, J=8.0 \mathrm{~Hz}$,
$\mathrm{CH}_{2}$ ), $3.76\left(\mathrm{dd}, 1 \mathrm{H}, J=16.0,8.0 \mathrm{~Hz}, \mathrm{NCH}_{2}\right.$ ), 3.57 (dd, $1 \mathrm{H}, J=16.0,8.0 \mathrm{~Hz}$, $\mathrm{NCH}_{2}$ ), 2.47 (s, 3H, C=CCH $), 1.09-1.01\left(\mathrm{~m}, 6 \mathrm{H}, \mathrm{OCH}_{2} \underline{\mathrm{CH}}_{3}, \mathrm{NCH}_{2} \underline{\mathrm{CH}}_{3}\right.$ ); ${ }^{13} \mathrm{C}$ NMR (100 MHz, DMSO- $d_{6}$ ) $\delta: 165.88,152.72,150.28,143.56,132.28$, 128.86, 128.44, 102.74, 60.03, 52.42, 37.40, 15.91, 15.20, 14.46; IR (KBr): 3413, 2985, 1686, 1616, $768 \mathrm{~cm}^{-1}$. Anal. calcd. for $\mathrm{C}_{16} \mathrm{H}_{19} \mathrm{~N}_{2} \mathrm{O}_{3} \mathrm{Cl}$ : C 59.54, H 5.93, N 8.68; found C 59.60, H 5.91, N 8.72.

2.2.5 5-Ethoxycarbonyl-1-ethyl-6-methyl-4-(4-nitrophenyl)-3,4dihydropyrimidin-2(1H)-one (q): Yellow solid. ${ }^{1} \mathrm{H}$ NMR $(400 \mathrm{MHz}$, DMSO- $\left.d_{6}\right) \delta: 8.18(\mathrm{~d}, 2 \mathrm{H}, J=8.0 \mathrm{~Hz}, \mathrm{ArH}), 8.05(\mathrm{~d}, 1 \mathrm{H}, J=4.0 \mathrm{~Hz}$, $\mathrm{NH}), 7.44$ (d, $2 \mathrm{H}, J=8.0 \mathrm{~Hz}, \mathrm{ArH}), 5.23(\mathrm{~d}, 1 \mathrm{H}, J=4.0 \mathrm{~Hz}, \mathrm{CH}), 4.00$ (q, $2 \mathrm{H}, J=8.0 \mathrm{~Hz}, \mathrm{CH}_{2}$ ), 3.76 (dd, $1 \mathrm{H}, J=16.0,8.0 \mathrm{~Hz}, \mathrm{NCH}_{2}$ ), 3.58 (dd, $\left.1 \mathrm{H}, J=16.0,8.0 \mathrm{~Hz}, \mathrm{NCH}_{2}\right), 2.48\left(\mathrm{~s}, 3 \mathrm{H}, \mathrm{C}=\mathrm{CCH}_{3}\right), 1.09-1.02(\mathrm{~m}, 6 \mathrm{H}$, $\left.\mathrm{OCH}_{2} \underline{\mathrm{CH}}_{3}, \mathrm{NCH}_{2} \underline{\mathrm{CH}}_{3}\right) ;{ }^{13} \mathrm{C}$ NMR (100 MHz, DMSO- $\left.d_{6}\right) \delta: 165.72,152.56$, 151.78, 151.02, 147.16, 127.88, 124.28, 102.02, 60.17, 52.63, 37.5215 .97 15.18 14.44; IR (KBr): 3405, 2985, 1692, 1616, $768 \mathrm{~cm}^{-1}$. Anal. calcd. for $\mathrm{C}_{16} \mathrm{H}_{19} \mathrm{~N}_{3} \mathrm{O}_{5}: \mathrm{C} 57.65$, H 5.74, N 12.60; found C 57.56, H 5.71, N 12.66.

2.2.6 5-Methoxycarbonyl-1-ethyl-6-methyl-4-(4-nitrophenyl)3,4-dihydropyrimidin-2(1H)-one (r): Pale yellow solid. ${ }^{1} \mathrm{H}$ NMR $\left(400 \mathrm{MHz}\right.$, DMSO- $\left.d_{6}\right) \delta: 8.22(\mathrm{~d}, 2 \mathrm{H}, J=8.0 \mathrm{~Hz}, \mathrm{ArH}), 8.12(\mathrm{~d}, 1 \mathrm{H}$, $J=4.0 \mathrm{~Hz}, \mathrm{NH}), 7.49$ (d, 2H, $J=8.0 \mathrm{~Hz}, \mathrm{ArH}), 5.26(\mathrm{~d}, 1 \mathrm{H}, J=4.0 \mathrm{~Hz}, \mathrm{CH}$ ), $3.81\left(\mathrm{dd}, 1 \mathrm{H}, J=16.0 \mathrm{~Hz}, 8.0 \mathrm{~Hz}, \mathrm{NCH}_{2}\right), 3.63(\mathrm{dd}, 1 \mathrm{H}, J=16.0,8.0 \mathrm{~Hz}$, $\left.\mathrm{NCH}_{2}\right), 3.58\left(\mathrm{~s}, 3 \mathrm{H}, \mathrm{OCH}_{3}\right), 2.53\left(\mathrm{~s}, 3 \mathrm{H}, \mathrm{C}=\mathrm{CCH}_{3}\right), 1.07(\mathrm{t}, 3 \mathrm{H}, J=8.0 \mathrm{~Hz}$, $\mathrm{NCH}_{2} \mathrm{CH}_{3}$ ); ${ }^{13} \mathrm{C}$ NMR (100 MHz, DMSO- $\left.d_{6}\right) \delta: 166.23,152.61,151.58$, 151.30, 147.18, 127.83, 124.31, 101.81, 52.44, 51.68, 37.54, 16.02, 15.18; IR (KBr): 3397, 2992, 1686, 1616, $768 \mathrm{~cm}^{-1}$. Anal. calcd. for $\mathrm{C}_{15} \mathrm{H}_{17} \mathrm{~N}_{3} \mathrm{O}_{5}$ : C 56.42, H 5.37, N 13.16; found C 56.35, H 5.41, N 13.12.

2.2.7 5-Methoxycarbonyl-1-ethyl-6-methyl-4-(2-chlorophenyl)3,4-dihydropyrimidin-2(1H)-one (s): Pale yellow solid. ${ }^{1} \mathrm{H}$ NMR $\left(400 \mathrm{MHz}\right.$, DMSO- $\left.d_{6}\right) \delta: 7.89(\mathrm{~d}, 1 \mathrm{H}, J=4.0 \mathrm{~Hz}, \mathrm{NH}), 7.42(\mathrm{~d}, 1 \mathrm{H}$, $J=8.0 \mathrm{~Hz}, \mathrm{ArH}$ ), 7.32-7.25 (m, 3H, ArH), 5.59 (s, 1H, CH), 3.83 (dd, 1H, $\left.J=16.0,8.0 \mathrm{~Hz}, \mathrm{NCH}_{2}\right), 3.65\left(\mathrm{dd}, 1 \mathrm{H}, J=16.0,8.0 \mathrm{~Hz}, \mathrm{NCH}_{2}\right), 3.48(\mathrm{~s}, 3 \mathrm{H}$, $\mathrm{OCH}_{3}$ ), 2.56 (s, 3H, $\mathrm{C}=\mathrm{CCH}_{3}$ ), 1.12 (t, 3H, $\left.J=8.0 \mathrm{~Hz}, \mathrm{NCH}_{2} \mathrm{CH}_{3}\right) ;{ }^{13} \mathrm{C} \mathrm{NMR}$ $\left(100 \mathrm{MHz}\right.$, DMSO- $\left.d_{6}\right) \delta: 166.21,152.12,151.07,141.21,132.16,130.02$, 129.70, 128.77, 128.15, 101.44, 51.50, 50.52, 37.45, 15.90, 15.23; IR (KBr): 3417, 2983, 1683, 1634, $768 \mathrm{~cm}^{-1}$. Anal. calcd. for $\mathrm{C}_{15} \mathrm{H}_{17} \mathrm{~N}_{2} \mathrm{O}_{3} \mathrm{Cl}$ : C 58.35, H 5.55, N 9.07; found C 58.42, H 5.60, N 9.01.

2.2.8 5-Ethoxycarbonyl-1-(4-methylphenyl)-6-methyl-4-phenyl3,4-dihydropyrimidin-2(1H)-one (t): Pale yellow solid. ${ }^{1} \mathrm{H}$ NMR $\left(400 \mathrm{MHz}, \mathrm{DMSO}-d_{6}\right) \delta: 8.18$ (s, $\left.1 \mathrm{H}, \mathrm{NH}\right), 7.12-7.43$ (m, 9H, ArH), 5.34 (s, $1 \mathrm{H}, \mathrm{CH}), 4.07$ (q, $\left.2 \mathrm{H}, J=4.0 \mathrm{~Hz}, \mathrm{OCH}_{2}\right), 2.36\left(\mathrm{~s}, 3 \mathrm{H}, \mathrm{C}=\mathrm{CCH}_{3}\right), 2.07$ (s, 3H, $\left.\mathrm{ArCH}_{3}\right), 1.14$ (t, 3H, $\left.J=4.0 \mathrm{~Hz}, \mathrm{CH}_{2} \mathrm{CH}_{3}\right) ;{ }^{13} \mathrm{C}$ NMR $\left(100 \mathrm{MHz}\right.$, DMSO- $\left.d_{6}\right)$ $\delta: 165.89,152.62,149.55,144.54,137.86,135.55,129.83,129.58,129.05$, 127.92, 126.71, 118.64, 60.14, 53.55, 21.10, 18.49, 14.46; IR (KBr): 3234, 2978, 1693, 1624, $758 \mathrm{~cm}^{-1}$. Anal. calcd. for $\mathrm{C}_{21} \mathrm{H}_{22} \mathrm{~N}_{2} \mathrm{O}_{3}: \mathrm{C} 71.98, \mathrm{H} 6.33$, N 7.99; found C 71.87, H 6.26, N 7.96.

2.2.9 5-Methoxycarbonyl-1-(4-methylphenyl)-6-methyl-4-phenyl3,4-dihydropyrimidin-2(1H)-one (u): Pale yellow solid. ${ }^{1} \mathrm{H}$ NMR $\left(400 \mathrm{MHz}, \mathrm{DMSO}-d_{6}\right) \delta: 8.21(\mathrm{~s}, 1 \mathrm{H}, \mathrm{NH}), 7.43-7.04(\mathrm{~m}, 9 \mathrm{H}, \mathrm{ArH})$, 5.34 (s, $1 \mathrm{H}, \mathrm{CH}), 3.61\left(\mathrm{~s}, 3 \mathrm{H}, \mathrm{OCH}_{3}\right), 2.35$ (s, 3H, C=CCH ), 2.08 (s, $\left.3 \mathrm{H}, \mathrm{ArCH}_{3}\right) ;{ }^{13} \mathrm{C}$ NMR (100 MHz, DMSO- $\left.d_{6}\right) \delta: 166.41,156.57,152.67$, 144.34, 137.90, 135.50, 129.85, 129.42, 129.12, 127.95, 126.64, 118.29, 53.36, 51.63, 21.09, 18.55; IR (KBr): 3430, 2922, 1693, 1654, $763 \mathrm{~cm}^{-1}$. Anal. calcd. for $\mathrm{C}_{20} \mathrm{H}_{20} \mathrm{~N}_{2} \mathrm{O}_{3}: \mathrm{C} 71.41, \mathrm{H} \mathrm{5.99}, \mathrm{N}$ 8.33; found C 71.33, H 6.02, N 8.37. 


\section{Results and discussion}

\subsection{Screening of catalysts}

First, we compared the catalytic activity of different metal methanesulfonates in Table 1. The activity of CMS is higher than that of the other methanesulfonates. 5-Ethoxycarbonyl-1,6-dimethyl-4-phenyl-3,4-dihydropyrimidin-2(1H)-one was obtained in 94\% yield within $0.3 \mathrm{~h}$ (entry 1). However, only $40 \%$ product yield was obtained in the absence of a catalyst (entry 14).

\subsection{Reaction temperature and reusable catalyst}

Then, we investigated the reaction temperature using the model reaction of benzaldehyde, ethyl acetoacetate, and methylurea. As shown in Table 2, the reaction at $90^{\circ} \mathrm{C}$ gave the best result (entry 3). When the reaction was completed, the reaction mixture was washed with water. The catalyst remained in aqueous phase and could be recovered by evaporating the filtrate. The CMS can be reused for the next two consecutive reactions. It demonstrated that CMS was water-tolerant and recyclable.

\subsection{One-pot synthesis of $N 1$-substituted DHPMs}

Next, N1-substituted DHPMs were synthesized under optimum conditions (Table 3). The Biginelli three-component reaction of aromatic aldehydes, $\beta$-ketoesters, and $N$-substituted (thio)ureas gave good yields of the products in a short time. The reaction accommodated a variety of aromatic aldehydes. Whether the substituents on the aromatic aldehyde are electron-withdrawing or -donating groups all afforded high yields. Unfortunately,
Table 2: Different reaction temperatures and the reusability of CMS.

\begin{tabular}{lrrr}
\hline Entry & Temperature $\left({ }^{\circ} \mathrm{C}\right)$ & Time (h) & Yield (\%) \\
\hline 1 & 70 & 0.7 & 93 \\
2 & 80 & 0.3 & 94 \\
3 & 90 & 0.2 & $96,93,89$ \\
\hline
\end{tabular}

Reaction conditions: benzaldehyde $(10 \mathrm{mmol})$, ethyl acetoacetate $(10 \mathrm{mmol})$, methylurea $(13 \mathrm{mmol}), \mathrm{CMS}(0.3 \mathrm{mmol})$, solvent-free.

substrates with aliphatic aldehydes gave no desired product. Ethyl acetoacetate and methyl acetoacetate were equally effective in carrying out the reaction. $N$-Methylurea, $N$-ethylurea, $p$-tolylurea, and $N$-methylthiourea were varied. In all cases, the pure products were obtained by simple filtration and recrystallization. No column chromatography or any cumbersome workup technique was applied. The methodology using a reusable catalyst without solvent is a green protocol obviously.

All the structures of the products were determined by IR, 1H NMR, 13C NMR, and elemental analysis. Furthermore, a single crystal detection of the product $\mathbf{t}$ was obtained to investigate the molecular structure (Figure 1). The pyrimidine ring composed of N1-C2-N3-C4-C5-C6 is a boat conformation. The $\mathrm{C}-4$ is a choral carbon and its configuration is R. An X-ray structure analysis verified the synthesized products.

\subsection{Reaction mechanism}

A possible reaction mechanism was proposed for this transformation [26]. At first, an aldehyde reacted with a (thio)urea to form an acylimine intermediate under the catalysis of CMS. Then, an open-chain ureide is obtained by the addition of a $\beta$-ketoester to the acylimine, which undergoes cyclization and dehydration to form the target product [Eq. (2)].

Table 1: Comparison of the catalytic activity among different metal methanesulfonates.

\begin{tabular}{|c|c|c|c|c|c|c|c|c|c|c|c|c|c|c|}
\hline Entry & 1 & 2 & 3 & 4 & 5 & 6 & 7 & 8 & 9 & 10 & 11 & 12 & 13 & 14 \\
\hline Metal & $\mathrm{Cu}$ & La & $\mathrm{Fe}(\mathrm{II})$ & $\mathrm{Zn}$ & $\operatorname{Pr}$ & $\mathrm{Yb}$ & $\mathrm{Ce}(\mathrm{III})$ & $\mathrm{Mg}$ & $\mathrm{Ni}$ & $\mathrm{Sr}$ & $\mathrm{Ca}$ & $\mathrm{Cd}$ & Al & - \\
\hline $\begin{array}{l}\text { Time } \\
\text { (h) }\end{array}$ & 0.3 & 0.4 & 0.7 & 0.3 & 0.3 & 0.5 & 1.0 & 1.5 & 1.0 & 2.5 & 1.5 & 1.3 & 2.0 & 3.0 \\
\hline $\begin{array}{l}\text { Yield } \\
(\%)\end{array}$ & 94 & 92 & 92 & 88 & 87 & 82 & 82 & 72 & 70 & 66 & 63 & 62 & 56 & 40 \\
\hline
\end{tabular}

Reaction conditions: benzaldehyde $(10 \mathrm{mmol})$, ethyl acetoacetate $(10 \mathrm{mmol})$, methylurea $(13 \mathrm{mmol})$, metal methanesulfonates $(0.3 \mathrm{mmol})$, solvent-free, $80^{\circ} \mathrm{C}$. 
Table 3: Three-component one-pot synthesis of N1-substituted DHPMs catalyzed by CMS.

\begin{tabular}{|c|c|c|c|c|c|c|c|}
\hline \multirow[t]{2}{*}{ Product } & \multirow[t]{2}{*}{$\mathbf{R}^{1}$} & \multirow[t]{2}{*}{$\mathbf{R}^{2}$} & \multirow[t]{2}{*}{$\mathbf{R}^{3}$} & \multirow[t]{2}{*}{$\mathbf{x}$} & \multirow[t]{2}{*}{ Yield (\%) (time/h) } & \multicolumn{2}{|r|}{$\mathrm{Mp}\left({ }^{\circ} \mathrm{C}\right)$} \\
\hline & & & & & & Found & Reported \\
\hline $\mathrm{a}$ & $\mathrm{H}$ & Et & $\mathrm{Me}$ & 0 & $96(0.2)$ & $178-180$ & $175-176[14]$ \\
\hline$b$ & $4-\mathrm{Cl}$ & Et & $\mathrm{Me}$ & 0 & $94(1.0)$ & $132-134$ & $133-135[14]$ \\
\hline c & 4- $\mathrm{CH}_{3}$ & Et & $\mathrm{Me}$ & 0 & $94(1.0)$ & $116-118$ & $119-120$ [14] \\
\hline d & $2-\mathrm{CH}_{3} \mathrm{O}$ & Et & $\mathrm{Me}$ & 0 & $89(0.8)$ & $147-149$ & $145-146[15]$ \\
\hline e & 4- $\mathrm{CH}_{3}^{3} \mathrm{O}$ & Et & $\mathrm{Me}$ & 0 & $93(1.5)$ & $138-140$ & $138-140[16]$ \\
\hline$f$ & $3-\mathrm{NO}_{2}$ & Et & $\mathrm{Me}$ & 0 & $92(0.5)$ & $137-139$ & $136-137[13]$ \\
\hline g & $4-\mathrm{OH}^{2}$ & Et & $\mathrm{Me}$ & 0 & $85(0.4)$ & $183-184$ & $178-180[16]$ \\
\hline h & $4-\mathrm{OH}-3-\mathrm{CH}_{3} \mathrm{O}$ & Et & $\mathrm{Me}$ & 0 & $94(0.3)$ & $181-183$ & - \\
\hline i & $\mathrm{H}$ & Et & $\mathrm{Me}$ & $\mathrm{S}$ & $40(6.0)$ & $145-147$ & $146-147[17]$ \\
\hline J & $\mathrm{H}$ & $\mathrm{Me}$ & $\mathrm{Me}$ & 0 & $94(0.2)$ & $193-195$ & 190-192 [16] \\
\hline k & $4-\mathrm{Cl}$ & $\mathrm{Me}$ & $\mathrm{Me}$ & 0 & $97(0.5)$ & $134-137$ & - \\
\hline l & $2-\mathrm{CH}_{3} \mathrm{O}$ & $\mathrm{Me}$ & $\mathrm{Me}$ & 0 & $78(0.2)$ & $171-173$ & $170-172[15]$ \\
\hline $\mathrm{m}$ & $3-\mathrm{OH}$ & $\mathrm{Me}$ & $\mathrm{Me}$ & 0 & $93(0.1)$ & $220-223$ & - \\
\hline$n$ & $\mathrm{H}$ & $\mathrm{Me}$ & $\mathrm{Me}$ & $\mathrm{S}$ & $46(6.0)$ & $159-160$ & $160-162[18]$ \\
\hline 0 & $\mathrm{H}$ & Et & Et & 0 & $63(3.0)$ & $112-115$ & $118[11]$ \\
\hline $\mathrm{p}$ & $4-\mathrm{Cl}$ & Et & Et & 0 & $93(2.0)$ & $146-148$ & - \\
\hline$q$ & $4-\mathrm{NO}_{2}$ & Et & Et & 0 & $92(1.5)$ & $152-154$ & - \\
\hline$r$ & $4-\mathrm{NO}_{2}$ & $\mathrm{Me}$ & Et & 0 & $94(0.8)$ & $198-200$ & - \\
\hline $\mathrm{s}$ & $2-\mathrm{Cl}^{2}$ & $\mathrm{Me}$ & Et & 0 & $70(1.5)$ & $121-123$ & - \\
\hline $\mathrm{t}$ & $\mathrm{H}$ & Et & $p-\mathrm{CH}_{3} \mathrm{C}_{6} \mathrm{H}_{4}$ & 0 & $82(3.0)$ & $179-181$ & - \\
\hline u & $\mathrm{H}$ & $\mathrm{Me}$ & $p-\mathrm{CH}_{3} \mathrm{C}_{6} \mathrm{H}_{4}$ & 0 & $95(3.0)$ & $152-158$ & - \\
\hline
\end{tabular}

Reaction conditions: aromatic aldehydes (10 mmol), $\beta$-ketoesters (10 mmol), $N$-substituted (thio)ureas (13 mmol), CMS (0.3 mmol), solvent-free, $90^{\circ} \mathrm{C}$.

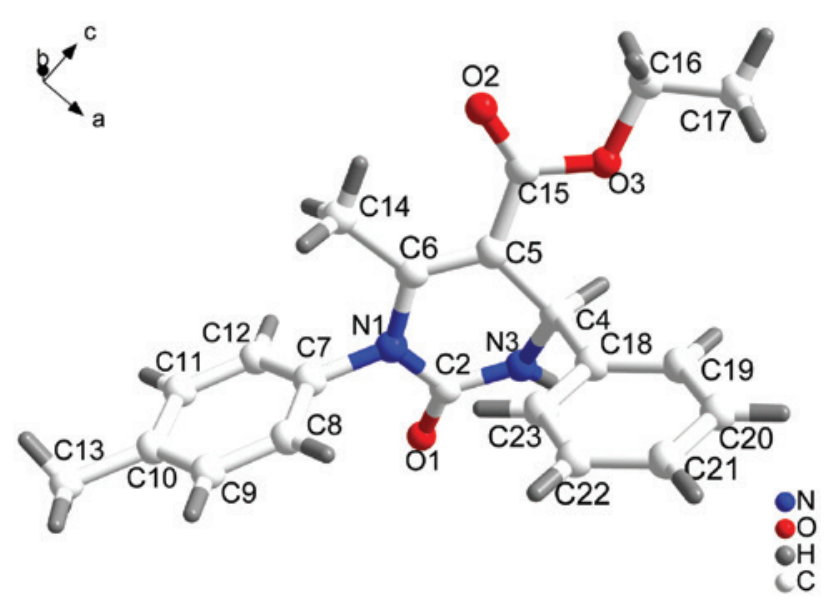

Figure 1: The molecular structure of the compound $t$.

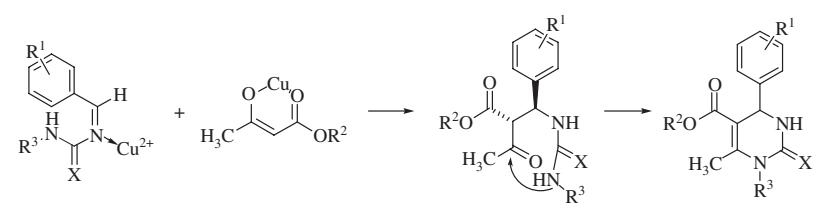

(2)

\section{Conclusions}

In conclusion, we have reported a simple, efficient, and environmentally friendly method to synthesize $N 1$-substituted DHPMs from aromatic aldehydes, ethyl acetoacetate/ methyl acetoacetate, and monosubstituted (thio)ureas with CMS as a catalyst at $90^{\circ} \mathrm{C}$. Neither toxic solvent nor expensive catalyst was used. It has mild reaction conditions, simple operation, short reaction time, high yield, and other environmentally friendly advantages. The most notable feature is the expansion of the substrates. The substituents on the urea were beyond just Me or Et. The reaction scope is fairly general with regard to various substrates. Many novel N1-substituted DHPMs were synthesized first. The catalyst can be reused several times. The green methodology provides a better alternative to the existing reports.

Acknowledgments: This research work was financially supported by the Program for Liaoning Excellent Talents in University (no. LJQ2015002), the Natural Science Foundation of Liaoning Province (no. 2015020201), and the National Natural Science Foundation of China (no. 21406016). 


\section{References}

[1] Atwal KS, Rovnyak GC, Kimball SD, Floyd DM, Moreland S, Swanson BN, Gougoutas JZ, Schwartz J, Smillie KM, Malley MF. J. Med. Chem. 1990, 33, 2629-2635.

[2] Atwal KS, Swanson BN, Unger SE, Floyd DM, Moreland S, Hedberg A, O’Reilly BC. J. Med. Chem. 1991, 34, 806-811.

[3] Rovnyak GC, Atwal KS, Hedberg A, Kimball SD, Moreland S, Gougoutas JZ, O’Reilly BC, Schwartz J, Malley MF. J. Med. Chem. 1992, 35, 3254-3263.

[4] Rovnyak GC, Kimball SD, Beyer B, Cucinotta G, DiMarco JD, Gougoutas J, Hedberg A, Malley M, McCarthy JP, Zhang R, Moreland S. J. Med. Chem. 1995, 38, 119-129.

[5] Cho H, Ueda M, Shima K, Mizuno A, Hayashimatsu M, Ohnaka Y, Takeuchi Y, Hamaguchi M, Aisaka K, Hidaka T, Kawai M, Takeda M, Ishihara T, Funahashi K, Satoh F, Morita M, Noguchi T. J. Med. Chem. 1989, 32, 2399-2406.

[6] Grover GJ, Dzwonczyk S, McMullen DM, Normandin DE, Parham CS, Sleph PG, Moreland S. J. Cardiovasc. Pharmacol. 1995, 26, 289-294.

[7] Cochran JC, Gatial JE, Kapoor TM, Gilbert SP. J. Biol. Chem. 2005, 280, 12658-12667.

[8] Kumarasamy D, Roy BG, Rocha-Pereira J, Neyts J, Nanjappan S, Maity S, Mookerjee M, Naesens L. Bioorg. Med. Chem. Lett. 2017, 27, 139-142.

[9] Terracciano S, Lauro G, Strocchia M, Fischer K, Werz O, Riccio R, Bruno I, Bifulco G. ACS Med. Chem. Lett. 2015, 6, 187-191.

[10] Patil AD, Kumar NV, Kokke WC, Bean MF, Freyer AJ, Brosse C, Mai S, Truneh A, Faulkner DJ, Carte B, Breen AL, Hertzberg RP,
Johnson RK, Westley JW, Potts BCM. J. Org. Chem. 1995, 60, 1182-1188.

[11] Singh K, Arora D, Poremsky E, Lowery J, Moreland RS. Eur. J. Med. Chem. 2009, 44, 1997-2001.

[12] Zalavadiya P, Tala S, Akbari J, Joshi H. Arch. Pharm. Chem. Life Sci. 2009, 342, 469-475.

[13] Putatunda S, Chakraborty S, Ghosh S, Nandi P, Chakraborty S, Sen PC, Chakraborty A. Eur. J. Med. Chem. 2012, 54, 223-231.

[14] Besoluk S, Kucukislamoglu M, Nebioglu M, Zengin M, Arslan M. J. Iran. Chem. Soc. 2008, 5, 62-66.

[15] Aridoss G, Jeong YT. Bull. Korean Chem. Soc. 2010, 31, 863-868.

[16] Salehi H, Guo QX. Chinese J. Chem. 2005, 23, 91-97.

[17] Kappe CO, Kumar D, Varma RS. Synthesis 1999, 1799-1803.

[18] Memarian HR, Ranjbar M. J. Chin. Chem. Soc. 2011, 58, 522-527.

[19] Lauro G, Strocchia M, Terracciano S, Bruno I, Fischer K, Pergola C, Werz O, Riccio R, Bifulco G. Eur. J. Med. Chem. 2014, 80, 407-415.

[20] Zlatković DB, Radulović NS. RSC Adv. 2016, 6, 115058-115067.

[21] Wipf P, Cunningham A. Tetrahedron Lett. 1995, 36, 7819-7822.

[22] Chen Q, Liu QJ, Wang HP. Molbank 2012, 2012, M752.

[23] Ryabukhin SV, Plaskon AS, Bondarenko SS, Ostapchuk EN, Grygorenko 00, Shishkin OV, Tolmachev AA. Tetrahedron Lett. 2010, 51, 4229-4232.

[24] Andleeb S, Imtiza D, Rauf MK, Azam SS, Badshah A, Sadaf H, Raheel A, Tahir MN, Raza S. RSC Adv. 2016, 6, 79651-79661.

[25] Wang M, Song ZG, Jiang H, Gong H. J. Therm. Anal. Calorim. 2009, 98, 801-806.

[26] Kappe CO. J. Org. Chem. 1997, 62, 7201-7204. 